\title{
ENDOTOXIN REMOVAL USING MICROMACHINED SILICON NANOPOROUS MEMBRANES
}

\author{
R.A. Smith ${ }^{1,2}$, K. Goldman ${ }^{3}$, A.J. Fleischman ${ }^{1}$, W.H. Fissell ${ }^{1}$, C.A. Zorman ${ }^{2}$, and S. Roy ${ }^{1}$ \\ ${ }^{1}$ Department of Biomedical Engineering, Cleveland Clinic, Cleveland, Ohio, USA \\ ${ }^{2}$ Dept. of Electrical Engineering and Computer Science, Case Western Reserve University, Cleveland, Ohio USA \\ ${ }^{3} \mathrm{H}$-Cubed, Inc., Cleveland, Ohio USA
}

\begin{abstract}
In this work, micromachined silicon nanoporous membranes (MSNM) are introduced as a means for removing endotoxin from water for medical uses. MSNM with critical pore dimensions of 35 $\mathrm{nm}$ and $42 \mathrm{~nm}$ and a $0.2 \mu \mathrm{m}$ syringe filter were challenged with feed solutions containing endotoxin activities of around 20,000 $\mathrm{EU} / \mathrm{mL}$. MSNM filters showed a reduction in endotoxin activities by 2 to 3 orders of magnitude.
\end{abstract}

\section{INTRODUCTION}

Endotoxins are are lipopolysacharide fragments from the outer cell membrane in Gram-negative bacteria that are known to cause septic shock, tissue injury, and death in organisms [1]. As such, it is critical for the endotoxin levels in medical solutions to be minimized. For intravenous (IV) use, the limit of acceptable endotoxin presence has been set by the United States at 0.25 endotoxin units (EU) per mL. Previous work in endotoxin removal has focused on methods based on size exclusion [2], charge exclusion [3], and adsorption [3-4]. Ceramic filters are attractive for endotoxin removal because of their ability to withstand the harsh environments employed during cleaning [5]. Ceramic filters generally have a wide pore size distribution based on their method of manufacture. With endotoxin levels of $1000 \mathrm{EU} / \mathrm{mL}$ in the feed solution, these filters are capable of reducing endotoxin activity in the filtrate to IV use specifications in dead-end filtration, but not when used in cross-flow filtration applications [5]. Cross-flow filtration is desirable for many applications because it generally extends filter lifetime by reducing filter fouling.

\section{DEVICE DESCRIPTION}

Micromachined silicon nanoporous membranes (MSNM) with monodispersed pore size distributions of the type shown in the schematic of Fig. 1 are an attractive alternative to ceramic filters for size-exclusion based endotoxin removal. Like ceramic membranes, MSNM are chemically, mechanically, and biologically stable [6]. The membranes are made of polysilicon films that are deposited on nitride-coated (100) Si wafers. Standard Si micromachining techniques combined with $\mathrm{Si}$ thermal oxidation can be used to produce nanopore membranes with very tight tolerances on pore dimensions and distribution; the critical dimensions of all pores varying by only 5\% [7]. MSNM are currently of interest as size-exclusion based filters for use in renal replacement applications [8]. Based on previous work in molecular sieving, we hypothesize that MSNM can deliver superior functionality and performance over conventional size-exclusion based filtration technologies since the pore geometries are more tightly controlled [9].

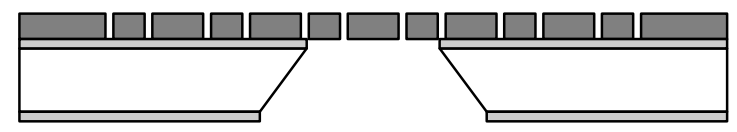

Figure 1: Cross-sectional schematic of a MSNM used in this study. Pores are formed by selective removal of a sacrificial oxide that is thermally grown on the sidewalls of a patterned polysilicon film.

\section{EXPERIMENTAL}

In this study, MSNMs of the type shown in Fig. 2 with pore sizes of $35 \mathrm{~nm}$ and $42 \mathrm{~nm}$ and a commercially-available $0.22 \mu \mathrm{L}$ syringe filter were evaluated for their effectiveness in endotoxin removal. Using the apparatus shown in Fig. 3, a mixture composed of $1 \mathrm{~mL}$ concentrated endotoxin solution and $100 \mathrm{~mL}$ of filtered DI water was circulated by a peristaltic pump in a closed circuit from the liquid reservoir past the MSNM chip in a cross-flow orientation. The filtrate was collected in a sterile microcenterfuge tube. For each filter, three $1 \mathrm{~mL}$ samples of filtrate and three $1 \mathrm{~mL}$ samples of the feed solution were collected, though only one feed sample for the syringe filter was tested for endotoxin activity. In addition, $1 \mathrm{~mL}$ samples of filtered DI water and endotoxin-free water were collected for comparison purposes. After collection, the samples were sent to Nelson Labs (Salt Lake City, UT) to determine endotoxin levels. The test employed for EU quantification was the LAL kinetic turbidimetric test which had a range of $0.005 \mathrm{EU} / \mathrm{mL}$ to $5 \mathrm{EU} / \mathrm{mL}$. If the filtrate had an endotoxin content greater than the available range, serial dilutions were performed until a reading could be made.
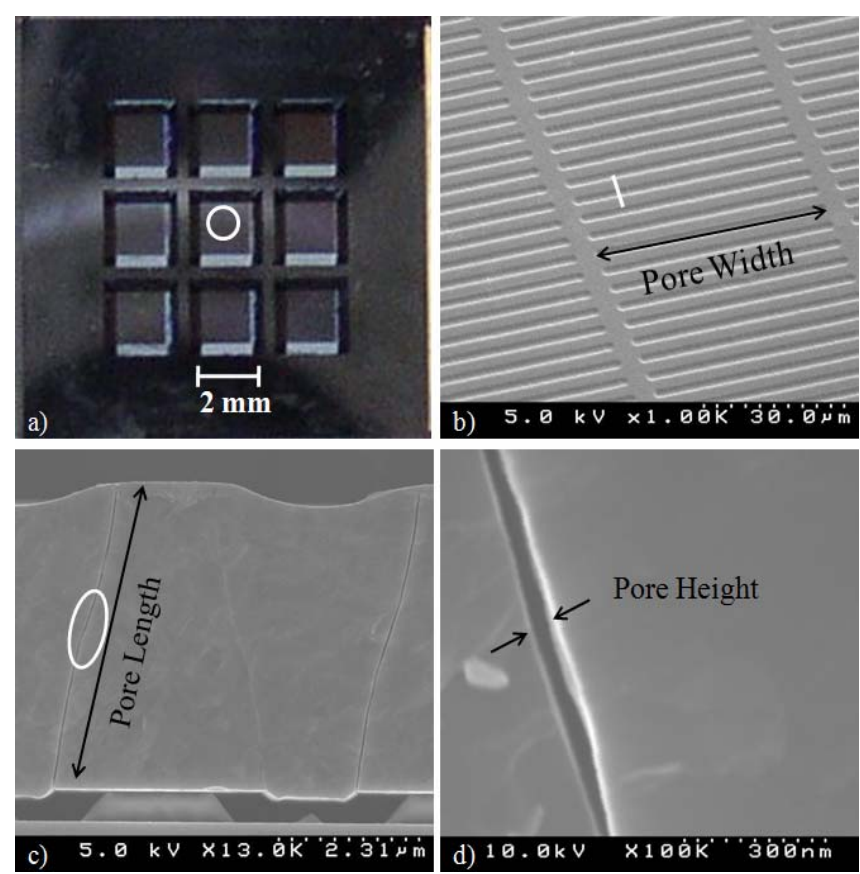

Figure 2: Images of a micromachined silicon nanoporous membrane chip: a) An optical image of an entire chip. b) An SEM of the top surface of the membrane which illustrates the width of the nanopores. c) A cross-sectional SEM of a nanoporous membrane which illustrates the length of the nanopores. d) A close-up cross sectional view showing an individual nanopore. At this magnification, the pore height (the critical dimension) can be measured (42 $\mathrm{nm}$ in this case). 


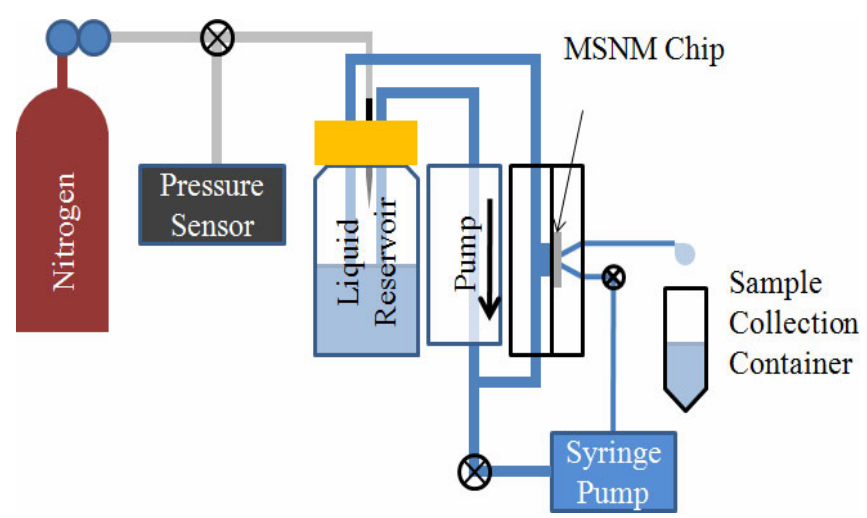

Figure 3: Schematic diagram of the experimental setup used to evaluate the MSNM filter chips. Pressure is applied to a liquid reservoir that contains the feed solution. The pressurized feed solution is circulated past the MSNM chip by a peristaltic pump. Filtrate is collected in a sterile microcenterfuge tube. The MSNM is mounted in a cross-flow filtration.

\section{RESULTS}

Table 1 summarizes the measured endotoxin levels for all samples. Both MSNM chips were capable of dramatically reducing the endotoxin levels, whereas the syringe filter did not appreciably reduce endotoxin levels. Although the endotoxin levels in the MSNM filtrate were not at levels suitable for IV use, the endotoxin levels in the feed solutions were more than 100x higher than those found in typical tap water $(125 \mathrm{EU} / \mathrm{mL})$ [10]. The extremely high endotoxin level in the feed solution was chosen to provide high contrast between the feed solution and filtrate. A comparison of endotoxin in the filtrate with that in the feed as a function of pore size is shown in Fig. 4. These data suggest that pore sizes below 30 $\mathrm{nm}$ are required to achieve filtrate that meets IV standards when feed stock with high endotoxin concentrations is used. MSNM with pore sizes below $10 \mathrm{~nm}$ are readily achievable and evaluation of these membranes is currently underway.

Table 1: Endotoxin levels in the feed solution and filtrate. Uncertainties for endotoxin level measurements are $+100 \% /-50 \%$.

\begin{tabular}{|c|c|}
\hline \multicolumn{2}{|c|}{ MSNM-35 } \\
\hline Feed (EU/mL) & Filtrate (EU/mL) \\
\hline 22000 & 390 \\
\hline 21000 & 15 \\
\hline 19000 & 9.7 \\
\hline \multicolumn{2}{|c|}{ MSNM-42 } \\
\hline Feed (EU/mL) & Filtrate (EU/mL) \\
\hline 23000 & 61 \\
\hline 25000 & 16 \\
\hline 23000 & Filtrate (EU/mL) \\
\hline \multicolumn{2}{|c|}{22000} \\
\hline Feed (EU/mL) & 17000 \\
\hline 24000 & 26000 \\
\hline \multicolumn{2}{|c|}{ Other Data } \\
\hline & Endotoxin-Free Water (EU/mL) \\
\hline & $<0.005$ \\
\hline DI Water (EU/mL) &
\end{tabular}

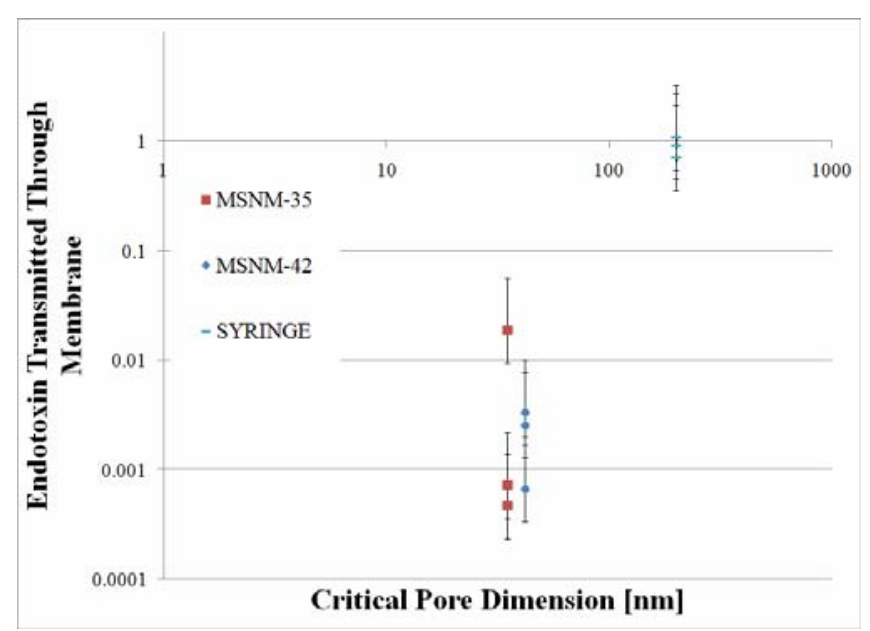

Figure 4: Measurement of endotoxin activity in the filtrate relative to endotoxin activity in the feed solution as a function of MSNM pore size. By comparison, a $0.22 \mu \mathrm{m}$ syringe filter did not exhibit any endotoxin rejection.

\section{ACKNOWLEDGEMENTS}

This work was made possible by NASA STTR Grant \# 06 T3.02-9924.

\section{REFERENCES}

[1] P. Magalhães, et al., "Methods of endotoxin removal from biological preparations: a review", Journal of Pharmaceutical Science, 10, 271-287 (2007).

[2] K.J. Sweander, M.Forte, and L. Nelson, "Filtration removal of endotoxin(pyrogens) in solution in different states of aggregation", Applied and Environmental Microbiology, 34, 382-385, (1977).

[3] C.P. Gerba and K. Hou, Kenneth. "Endotoxin removal by charge-modified filters" Applied and Environmental Microbiology, 50, 1375-1377, (1985).

[4] R.L. Machado, et al., "Evaluation of a chitosan membrane for removal of endotoxin from human IgG solutions" Process Biochemistry, 41, 2252-2257, (2006).

[5] P. Czermak, M. Ebrahimi, and G. Catapano. "New generation ceramic membranes have the potential of removing endotoxins from dialysis water and dialysate", International Journal of Artificial Organs, 28, 694-700 (2005).

[6] F.J. Martin, and C. Grove, "Microfabricated Drug Delivery Systems: Concepts to Improve Clinical Benefit" Biomedical Microdevices, 3, 97-108, (2001).

[7] T.A. Desai, D.J. Hansford, and M. Ferrari, "Micromachined Interface: New Approaches in Cell Immunoisolation", Biomolecular Engineering, 17, 23-26 (2000).

[8] W.H. Fissell, et al., "Dialysis and Nanotechnology: Now, 10 Years, or Never?”, Blood Purification, 25, 12-17, (2007)

[9] J. Han, J. Fu, and R.B. Schoch, "Molecular sieving using nanofilters: Past, present and future”, Lab on a Chip, 8, 23-33 (2008)

[10] W.B. Anderson, R.M. Slawson, and C.I. Mayfield, “A review of drinking-water-associated endotoxin, including potential routes to human exposure", Canadian Journal of Microbiology, 48, 567-587 (2002). 